\title{
Fehler- und Strafpraktiken am Ende der Frühen Neuzeit am Beispiel von Schule und Unterricht in der Deutschschweiz
}

\author{
Horlacher, Rebekka
}

DOI: https://doi.org/10.14361/9783839453742-008

Posted at the Zurich Open Repository and Archive, University of Zurich ZORA URL: https://doi.org/10.5167/uzh-196049

Book Section

Published Version

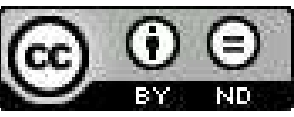

The following work is licensed under a Creative Commons: Attribution-NoDerivatives 4.0 International (CC BY-ND 4.0) License.

Originally published at:

Horlacher, Rebekka (2020). Fehler- und Strafpraktiken am Ende der Frühen Neuzeit am Beispiel von Schule und Unterricht in der Deutschschweiz. In: Hoffmann-Ocon, Andreas; De Vincenti, Andrea; Grube, Norbert. Praxeologie in der Historischen Bildungsforschung: Möglichkeiten und Grenzen eines Forschungsansatzes. Bielefeld, Germany: Transcript Verlag, 243-268.

DOI: https://doi.org/10.14361/9783839453742-008 


\section{Fehler- und Strafpraktiken am Ende der Frühen Neuzeit am Beispiel von Schule und Unterricht in der Deutschschweiz}

Rebekka Horlacher

Der mahnende Zeigefinger von Wilhelm Buschs Lehrer Lämpel ist aus der Ikonografie des Schulmeisters nicht wegzudenken, dies zumindest im deutschsprachigen Raum. Die Körpersprache der auf dieser Zeichnung skizzierten Figur mahnt nicht nur eine unsichtbare Schülerschaft zur Aufmerksamkeit, sondern impliziert auch deren potenzielles Fehlverhalten sowie die entsprechende Bestrafung. Der Lehrer, so der Eindruck, der durch dieses Bild hervorgerufen wird, ist nicht nur ein Vermittler von Wissen, sondern auch für das richtige Verhalten zuständig, was - im Wissen um die Fortsetzung der Geschichte von >Max und Moritz‘, die in der Getreidemühle und als Entenfutter enden - nicht ohne eine bestimmte Form des Strafens möglich zu sein scheint. Solche oder ähnliche Überlegungen könnten Busch zu seiner Zeichnung motiviert haben und sie können auch als Hinweise auf ein bestimmtes Bild des Lehrers verstanden werden. Sie sind aber keine Aussagen darüber, wie sich die Lehrer um 1865, dem Jahr der Erstpublikation von >Max und Moritz , in ihrem Unterrichtsalltag verhalten haben, noch bietet die Zeichnung ein Abbild einer konkreten Unterrichtspraxis oder der damit verbundenen gesellschaftlichen Normvorstellungen. Es ist vielmehr die zeichnerische Darstellung einer Lehrerfigur, die historische Vorbilder haben kann, aber nicht haben muss und die eine bestimmte Praxis spiegeln möchte, diese in der Darstellung aber auch ironisieren oder verfremden kann.

Die Differenz von Bild - oder Text - zur historischen »Wirklichkeit« ist ein wiederkehrendes Thema der Historiografie, das viele verschiedene Antworten generiert hat, die aber alle keinen abschliessenden Wahrheitsgehalt für sich beanspruchen können. Das »Problem« von Fakten und deren Interpretation bleibt als epistemologisches Thema konstant. ${ }^{1}$ Die Frage, inwiefern Texte als Beschreibung oder Abbild von Praxis gelesen werden können, beschäftigt die Kulturgeschichte seit dem 19. Jahrhundert. Da in dieser Tradition nicht politische Handlungen, Gesetzgebungen, statistische Daten oder philosophische Konzepte im Fokus des 
Forschungsinteresses stehen, sondern Bräuche, Sitten oder Gewohnheiten, die sich in Tätigkeiten ausdrücken, erfuhren diese Fragen besondere Aufmerksamkeit, wobei sich diese Debatten spätestens seit den 1970er-Jahren und mit dem Aufkommen der Alltagsgeschichte, der Mikrogeschichte oder der Historischen Anthropologie deutlich intensiviert haben. ${ }^{2}$ Ohne hier näher auf die unterschiedlichen Traditionen von Kulturgeschichte einzugehen, die eng mit den verschiedenen Kulturbegriffen im deutsch-, französisch- oder englischsprachigen Kontext zusammenhängen, ${ }^{3}$ kann festgehalten werden, dass der mit diesen historiografischen Entwicklungen verbundene cultural turn in der Geschichtswissenschaft den Blick der Forschenden verstärkt auf die materielle Basis der Vergangenheit und auf verschiedene Formen von »Praxis« gelenkt hat, wobei jeweils sehr unterschiedlich ausfällt, welche Aspekte berücksichtigt werden.

Eine dieser Möglichkeiten ist die Beschäftigung mit Praktiken, die hier als »Verhaltens- und Handlungsweisen in ihren historisch-sozialen Zusammenhängen « verstanden werden. ${ }^{4}$ Praktiken bezeichnen Tätigkeiten, deren Beschreibungen, Begründungen und Legitimationen. Mit dem Einbezug der Begründungsund Legitimationsebene ist immer auch die Frage nach der Norm verbunden, wobei sich diese »auf verschiedenen Ebenen abspielen [kann], die viel mehr repräsentieren als allein obrigkeitliche Ordnungen und deren Befolgung oder Nichtbefolgung «. ${ }^{5}$ Die Norm ist keine Vorgabe, die Auswirkungen auf Praxis hat und der gefolgt oder die verletzt werden kann. Zum einen fliesst Praxis ebenso in die Norm mit ein und bestimmt diese mit, zum andern kann auch eine institutionalisierte Praxis, die breite gesellschaftliche Akzeptanz geniesst, normative Züge aufweisen, auch wenn sie gesetzlich nicht kodifiziert ist. Norm und Praxis sind somit keine statischen Konzepte, sondern entstehen im Austausch, und in Wechselwirkung. ${ }^{6}$ Bei der Diskussion um das Verhältnis von Norm und Praxis geht es denn auch nicht um die Frage von »Einfluss«, sondern um die Berücksichtigung der gegenseitigen Bedingtheiten vor dem Hintergrund der epistemologischen Frage, was »gemeint« ist, wenn etwas "gesagt« wird bzw. getan wurde. ${ }^{7}$

2 Wohl nicht zufällig waren es vor allem Frühneuzeitforscherinnen und -forscher, die in diesen Fragen besonders innovative Ideen in die Diskussion einbrachten, wird doch bei der Beschäftigung mit der Frühen Neuzeit die Differenz zur Gegenwart und die kulturelle »Andersheit« der untersuchten Zeit besonders offenkundig (Schmidt/Aspelmeier 2006, S. 8). Dies etwa im Unterschied zu Forschungen im 19. oder 20. Jahrhundert, die sich einfacherwenn auch nicht weniger unzutreffend - aus einer präsentistischen Perspektive und als "Vorgeschichte«der Gegenwart konzipieren lassen.

3 Vgl. Dinges 2002.

4 Mitterauer/Saurer 1993, S. 1.

5 Jaritz 1997, S. 18.

6 Alkemeyer/Buschmann/Michaeler 2015, S. $25 f$.

7 Vgl. Skinner 2002. 
Norm entsteht zudem nicht ausserhalb und unabhängig von Praxis, sie ist nicht göttlich oder natürlich, sondern sozial konstruiert und damit immer auch Teil der Praktik. "Eine von Normen unabhängige Praxis ist mithin ebenso wenig vorstellbar, wie sie überdies abseits einer - ausformulierten oder unreflektiert implizit unterstellten - >Theorie der Praxis beobachtet werden kann ${ }^{8}{ }^{8}$ Damit wird hier nicht ein Verständnis von Praxeologie in den Mittelpunkt gestellt, wie es vor allem in den 1970er- und 1980er-Jahren als Entwurf einer Allgemeinen Pädagogik diskutiert worden ist. Josef Derbolav beispielsweise formulierte eine Theorie der Pädagogik als Praxeologie, die sich als Handlungswissenschaft verstand und ethische Fragen ins Zentrum rückte. Ethik wurde dabei im Rückgriff auf einen aristotelischen Praxisbegriff als Verantwortungsethik konzipiert. ${ }^{9}$ Im Mittelpunkt steht vielmehr eine Praktik von Beschreibung und Reflexion konkreter Erfahrungen oder vorgestellter Handlungen, die auch als "normale Praxis $\aleph^{10}$ bezeichnet worden ist, wobei durch den Fokus auf Praktiken versucht wird, den "historischen Diskurs « der Praxis zu rekonstruieren. ${ }^{11}$

Am Beispiel von Überlegungen und konkreten Anweisungen zur Funktion und Prävention von und zum Umgang mit Fehlern und Strafen in verschiedenen »Leitfäden für Schulmeister « aus den letzten Dekaden des 18. Jahrhunderts sowie anhand von Bemerkungen zu Fehlern und Strafen in der Stapfer-Enquête wird hier danach gefragt, welche Praktiken des Fehlers und der Strafe sich im Lehreralltag am Ende des 18. Jahrhunderts zeigen. Die hier zum einen als Quellen dienenden »Leitfäden«, die in der zweiten Hälfte des 18. Jahrhunderts weit verbreitet waren, sind für diese Fragestellung besonders geeignet, weil sie als didaktisches Hilfsmittel und konkrete Unterstützung der Arbeit der Schulmeister gedacht waren. ${ }^{12}$ Ziel dieser Publikationen war es, den Lehrpersonen eine Anleitung und

9 Vgl. Derbolav 1975. An Derbolavs Überlegungen zur »Cesamtpraxis« schloss etwa Dietrich Benner in seiner Allgemeinen Pädagogik (1987) an (vgl. Müller 1995).

10 Dross 2006, S. 220.

11 Die deutschsprachige Bildungsgeschichte hat sich im Unterschied zur amerikanischen Forschung (vgl. z.B. Cuban 1984) lange Zeit eher schwer damit getan, anstelle von Ideen, Strukturen, Institutionen oder politischen Prozessen die Materialität und die Praxis von Erziehungsprozessen in den Blick zu nehmen (Depaepe 2000a, S. 10; vgl. Depaepe 2000b, S. 45f.). Erst in den letzten Jahren ist ein deutlicher Anstieg an entsprechender Forschung sichtbar geworden (vgl. z.B. Priem/König/Casale 2012).

12 Nicht berücksichtigt werden hier die verschiedenen »Landschulbibliotheken«, die zwar ein ähnliches Ziel verfolgten, bei denen es sich aber in der Regel um umfangreiche, mehrbändige Sammelwerke handelt, die nicht nur praktische Anleitungen zum Unterrichten boten, sondern sich auch auf theoretischer Ebene mit Schule, Unterricht und Erziehung befassten und die vor allem als regelmässig erscheinende Publikationen konzipiert waren. Damit boten sie eine fortlaufende Weiterbildungsmöglichkeit für aktive Lehrpersonen und beabsichtigten, das »neueste« pädagogische Wissen zu den Schulmeistern zu bringen (vgl. z.B. 
ein Hilfsmittel für ihre Berufstätigkeit zur Verfügung zu stellen, das nicht auf einer abstrakt-theoretischen Ebene Fragen von Erziehung, Bildung, Schule und Unterricht diskutierte. Vielmehr beanspruchten diese Publikationen - durchaus in einem (volks-)aufklärerischen Selbstverständnis - eine "nützliche« Unterstützung bei der organisatorischen und methodisch-didaktischen Bewältigung des Schulmeister-Alltags zu bieten. Damit war auch die Absicht verbunden, die Qualität der bestehenden Schule und des Unterrichts zu verbessern, ohne diese Qualitätsverbesserungen von Schulreformen oder anderen institutionellen Veränderungen abhängig machen zu müssen.

Als zweite Quelle dient die sogenannte Stapfer-Enquête, eine breit angelegte Schulumfrage, die der damalige helvetische Bildungsminister Philipp Albert Stapfer (1766-1840) 1799 veranlasst hatte, um auf einer empirisch soliden Basis die Schule reformieren und den neuen (politischen) Anforderungen anpassen zu können. ${ }^{13}$ Da sich der Fragebogen vor allem auf die organisatorischen Belange von Schule und Unterricht konzentriert, ist die Rubrik »Bemerkungen« für die hier verfolgte Fragestellung von besonderem Interesse. Die Lehrpersonen hatten dort die Möglichkeit, ihre persönlichen Fragen, Anregungen oder Überlegungen zu Schule und Unterricht zu notieren, und konnten so der helvetischen Regierung Vorschläge machen, wo und wie aus ihrer Sicht Schule und Unterricht zu reformieren waren.

Mit dieser Quellengrundlage wird nicht eine konkrete Strafpraxis als Reaktion auf fehlerhaftes Verhalten in den Blick genommen, ${ }^{14}$ sondern vielmehr das Reden bzw. Schreiben von einzelnen, teilweise auch als Schulmeister praktisch tätigen Personen auf das Themenfeld von Fehlern und Strafen. Da sich vor allem die Verfasser der Leitfäden nicht als beliebige Einzelpersonen verstanden, die ihr Nachdenken über schulisches, methodisches und pädagogisches Handeln zufälligerweise aufgeschrieben hatten, sondern ihre diesbezüglichen Publikationen als für die praktische Tätigkeit hilfreich und handlungsanleitend einschätzten, zeigt sich hier die Praktik des Strafens in dem Sinn, als dass die Beschreibung der Strafpraxis immer auch mit Begründungen versehen sind. ${ }^{15}$ Damit kommt diesen Quellen auch eine normierende Funktion zu, da sie auch zeigen, »wie Eliten - übrigens ganz verschiedener Art [...] - ein Stück der Wirklichkeit, in der sie lebten, verstanden und $\mathrm{zu}$ verstehen gaben«, wobei »diese Abbildungen des Sozialen [...] keine neutralen Reden« sind. "Sie erzeugen [vielmehr] Strategien und [...] Prak-

Johann Friedrich Prenningers Landschulbibliothek. Oder Handbuch für Schullehrer auf dem Lande. 4 Bde. [1780-1790] oder Johann Rudolf Steinmüllers Helvetische Schulmeister-Bibliothek 2 Bde. [1801]).

13 Vgl. Holenstein 2014.

14 Vgl.z.B. Herman u.a. 2003.

15 Dinges 1997, S. 41; Schmidt/Aspelmeier 2006, S. 8. 
tiken, die Autorität beanspruchen - und zwar auf Kosten anderer, denen sie abgesprochen « werden. ${ }^{16}$ Dabei ist interessant zu vergleichen - und dazu dienen die Bemerkungen in der Stapfer-Enquête -, inwiefern sich die in diesen "Leitfäden" geäusserten Überlegungen zu Fehlern und Strafen von den Aussagen der Schulmeister unterscheiden, die - aus welchen Gründen auch immer - dieses Themenfeld in ihren Antworten auf die Stapfer-Umfrage angesprochen haben. Allerdings ist auch hier festzuhalten, dass sich diese Schulmeister durch das Anfügen von Bemerkungen ebenfalls von der Mehrheit der "schweigenden« Schulmeister abhoben und damit in dem Sinne als »elitär« zu verstehen sind, als dass sie das Thema als diskussionswürdig erachteten.

In den ersten drei der insgesamt fünf Abschnitte dieses Beitrags werden fünf "Leitfäden « vorgestellt und auf ihre Aussagen zu den Themenfeldern von Fehlern und Strafen hin untersucht, wobei Publikationen ausgewählt wurden, die in der Schweiz im Umlauf waren, was ein Vergleich zu den Aussagen in der Stapfer-Enquête möglich macht, da sich die verschiedenen Quellen auf den gleichen Kontext beziehen bzw. von den Zeitgenossen als für den gleichen Kontext als passend angesehen wurden. Zunächst werden die beiden Anleitungen von Gottfried Christian Brünings (1727-1793) und Emanuel Merian (1732-1818) diskutiert, die beide an Basler Schulen verwendet wurden. Anschliessend werden die beiden »katholischen« Methodenbücher vorgestellt, das »Original « von Johann Ignaz von Felbiger (1724-1788) und die Schweizer Adaptation von Nivard Crauer (1747-1799), die in der Schweiz vor allem im Kanton Solothurn verbreitet waren. ${ }^{17}$ Der dritte Abschnitt beschäftigt sich mit den Bemerkungen von Johannes Büel (1761-1830), die hauptsächlich in der Region Zürich in Gebrauch waren und die mit ihren nummerierten »Merksätzen« eine von den anderen Handreichungen etwas unterschiedliche Form aufweisen. Der vierte Abschnitt beleuchtet das Thema Fehler und Strafe aus dem Blickwinkel der Schulmeister, die sich in der Stapfer-Enquête zu dieser Frage geäussert haben. Dabei zeigen sich grosse Übereinstimmungen zu den in den »Leitfäden« diskutierten Überlegungen, auch wenn sich nur sehr wenige Schulmeister zu diesem Themenkomplex geäussert haben. Im abschliessenden Abschnitt werden die Resultate zusammengefasst und die Frage diskutiert, ob und wenn ja welche Praktiken des Fehlers und des Strafens sich aus den Leitfäden und Bemerkungen in der Stapfer-Enquête rekonstruieren lassen, womit auch die Frage nach dem historiografischen »Mehrwert« einer solchen Perspektive diskutiert wird.

16 Chartier 1989, S. 12.

17 Vgl. Horlacher 2018. 


\section{Brünings' und Merians Anleitungen für Schulmeister}

1778 veröffentlichte der zu dieser Zeit in Heidelberg tätige und im kurpfälzischen Kreuznach geborene Pfarrer Brünings in Mannheim eine sAnleitung zu fruchtbarer Lehrart in den niedern Schulen<, die auf Veranlassung der Basler Aufmunterungsgesellschaft ${ }^{18}$ den Schulmeistern in Basel verteilt wurde. Brünings stellte im Vorwort der Anleitung seine Überlegungen als Resultat seiner bisherigen Arbeit als Beauftragter für die Vorbereitung der »Schul-Expektanten meines Vaterlandes « dar ${ }^{19}$ und wies darauf hin, dass sie »Resultat des ernstlichen Nachdenkens« seien, »durch welches ich den besten Weg, den niedern Schulen eine nutzbare und empfehlende Einrichtung zu geben, zu erforschen getrachtet habe ${ }^{20}$ Zudem betonte er, dass es sich bei diesen Überlegungen nicht um eine "Sammlung aus andern Erziehungsschriften ${ }^{21}$ handle, sondern um Überlegungen, die aus eigener Erfahrung stammten; ${ }^{22}$ ein in allen Anleitungen wiederkehrendes Argument, das jeweils die Qualität und die Nützlichkeit der eigenen Publikation betonen sollte.

Brünings Schrift stiess in Basel auf Kritik, da sie nicht das richtige Zielpublikum vor Augen habe. Bei den Basler Lehrern handle es sich in der Regel um "unstudierte Landleute«, weshalb er, der »würdige Vorsteher der baselischen Kirchen « Emanuel Merian, beschlossen habe, selber »eine solche [Anleitung] zu verfertigen, die den Bedürfnissen und den Fähigkeiten derer besonders angemessen wäre, welche die Gesellschaft diese Wohlthat zugedacht hatte.«In Merians Augen war die Brünings'sche Anleitung zu schwierig, zu komplex und zu umfangreich, um den Landschullehrern die gewünschte Hilfestellung zu leisten. In Merians Anleitung für die Landschulmeister (1778) dagegen würden die Schulmeister - so ist zumindest in einer Besprechung in Isaak Iselins Ephemeriden der Menschheit zu lesen -, »in dem wahren Tone eines Vaters und eines Hirten [...] von der Absicht ihres wichtigen Berufes « belehrt und mit denjenigen Mitteln ausgestattet, die sie für eine erfolgreiche Berufstätigkeit benötigten. ${ }^{23}$

Trotz dieser unterschiedlichen zeitgenössischen Wahrnehmung, was die Qualität und die Zielgruppenangemessenheit der beiden Anleitungen betrifft, unterscheiden sie sich nicht wesentlich in ihren Absichten, sie differieren allerdings in der Länge (Brünings: 128 Seiten, Merian: 63 Seiten) und in der inhaltlichen Breite.

18 Die Basler Aufmunterungsgesellschaft widmete sich der Förderung der Ausbildung der Bevölkerung und der Bekämpfung der Armut und war eine Vorläufergesellschaft der heutigen Gesellschaft für das Gute und Gemeinnützige (GCG). 
Während Brünings die »klassischen Unterrichtsfächer« Lesen, Rechnen, Schreiben, Singen, Katechismus jeweils in Bezug auf ihre methodische Umsetzung diskutierte und diesen Abschnitten einige grundsätzliche Kapitel zur Schule und zur Aufgabe des Schulmeisters voranstellte, widmete Merian den grössten Teil seiner Abhandlung nicht den Unterrichtsfächern, sondern der Schule und den Aufgaben des Schulmeisters. Methodische Anleitungen für den Unterricht scheinen in Merians Augen offenbar kein Bedürfnis der Basler Schulmeister darzustellen, Ratschläge und Leitlinien für das richtige Verständnis von Schule und dem dazugehörigen beruflichen Selbstverständnis allerdings schon. Einer dieser Abschnitte war - dies sowohl bei Brünings als auch bei Merian - der »Schulzucht« gewidmet und damit der Frage nach dem pädagogischen Auftrag von Unterricht und Schule, was in diesen Handreichungen immer im Kontext der Überlegungen zu Fehlern und Strafen diskutiert wurde.

In Brünings Überlegungen, die mit »Rathgebungen wegen der Schulzucht» überschrieben sind, wird verschiedentlich deutlich, dass - dies durchaus in Übereinstimmung mit den zeitgenössischen Schulordnungen ${ }^{24}$ - das Strafen nicht der Normalfall, sondern die Ausnahme sein soll. ${ }^{25}$ Strafen waren in seinen Augen keine »normale« erzieherische Tätigkeit, sondern nur sehr gezielt, in bestimmten Fällen und mit einer spezifischen Absicht anzuwenden, wobei immer darauf zu achten sei, dass diese Tätigkeit die »Erlernung und Ausübung guter Sitten« befördere. ${ }^{26}$ Strafen waren zudem nicht zur Beschämung der Kinder einzusetzen ${ }^{27}$ oder um »knechtisches « Verhalten hervorzurufen, ${ }^{28}$ sondern zielten darauf $a b$, sich »überflüssig« zu machen, »die Kinder lenksam und gesittet« und »die Schulen zu einem eben so angenehmen als nützlichen Aufenthalt für die Kinder« werden zu lassen. ${ }^{29}$

Strafen sanktionierten bei Brünings allerdings nicht ausschliesslich moralisches Fehlverhalten, sondern dienten auch dazu, das Einüben der »klassischen« Sekundärtugenden wie Pünktlichkeit, Anstand und ein gepflegtes Erscheinen $\mathrm{zu}$ fördern, wobei Letzteres vor allem in einer Art »Sauberkeitserziehung « bestand und die Durchsetzung von hygienischen Standards unterstützte. ${ }^{30}$ Sowohl Pünktlichkeit als auch Anstand und gepflegtes Erscheinen waren aber nicht nur »äußere« Merkmale und Erziehungsziele, sondern bestimmten in Brünings' Vorstellung ganz entscheidend die moralische Erziehung mit. Strafen beförderten

\footnotetext{
24 Vgl. Schulordnung Basel 1766, S. $103 f$.

25 Brünings 1778, S. 111f. u. 115.

26 Ebd., S. 110.

27 Ebd., S. 114.

28 Ebd., S. 115.

29 Ebd., S. 116.

30 Ebd., S. 108.
} 
damit die Umsetzung von als »richtig« oder "gut« eingeschätzten Normvorstellungen, wobei in den Anleitungen nicht eine »theoretische« Norm formuliert wurde, der dann die eigene Strafpraxis zu folgen hatte. Es wurde im Gegenteil auf die eigene Erfahrung und damit die Praxis des Verfassers verwiesen und diese als Norm gesetzt, wobei auch die Gefahren einer fehlerhaften Anwendung diskutiert wurden.

Für Brünings mussten Strafen den gefehlt habenden Kindern einsichtig und nachvollziehbar gemacht werden, weshalb sie nicht leichtfertig anzudrohen und durchzuführen waren, ganz im Gegenteil: »Die angedrohete Strafen müssen auf eine feyerliche Art vollzogen werden $« .{ }^{31}$ Dem Strafen kam damit, ähnlich wie einer Belohnung, ein ritueller Aspekt zu, wobei es auch darum ging, die Kinder bzw. die Schülerinnen und Schüler in einen »strafempfänglichen« Zustand zu bringen. Die Herstellung dieses Zustandes betraf allerdings nicht nur die zu Bestrafenden, sondern auch den Schulmeister als strafende Person, der Strafen nicht im Affekt aussprechen oder gar ausführen sollte. Vielmehr war er angehalten, immer wieder von Neuem darauf hinzuweisen, »dass er in der Nothwendigkeit sey zu strafen, so ungern er es auch thut «, ${ }^{32}$ womit der Anschein vermieden werden konnte, »als ob er aus persönlicher Feindschaft, ungerecht, im Zorne, oder doch übereilt strafe $;^{33}$ alles Handlungen, die als unpassend bzw. als nicht den Normvorstellungen entsprechend abqualifiziert wurden.

Während bei Brünings die Überlegungen zur Strafe innerhalb des Kapitels "Rathgebungen wegen der Schulzucht» diskutiert wurden, stellte Merian seine Anmerkungen unter den Titel »Von den Züchtigungen, was dabei zu beobachten ist «. ${ }^{34}$ Im Unterschied zu Brünings konstatierte Merian gleich zu Beginn, dass die Ausübung der »Strafzucht « in der Schule »unumgänglich nothwendig« sei. ${ }^{35}$ Denn: »Die Bosheit, die sich bey der Jugend zeiget, und ohne Ahndung und Züchtigung zu ihrem grösten Verderben bey derselben überhand nehmen würde, macht die Strafe unentbehrlich «. ${ }^{36}$ Es war für Merian nicht die Frage, ob und wenn ja wie Strafe allenfalls notwendig sei. Die Notwendigkeit der Strafe wurde als natürlich bzw. göttlich gegeben angesehen, was mit einem Zitat Salomons aus den Sprüchen unterstützt wurde: "Laß nicht $a b$, den Knaben zu züchtigen; denn wenn $d u$ ihn mit der Ruthe hauest, so darfman ihn nicht tödten, sagt Salomo, Sprüchw. 23,13 «. ${ }^{37}$ Hingegen wurde auch bei Merian die »pädagogische Anwendung« der Strafe be-

31 Ebd., S. 111.

32 Ebd.

33 Ebd.

34 Merian 1779, S. 4.

35 Ebd., S. 46.

36 Ebd., S. $46 f$.

37 Ebd., S. 47. 
tont, da Strafen nur dann sinnvoll seien, wenn sie sowohl den Bestraften als auch die »Zuschauenden« davon abhalten würden, die bestrafte Tat ebenfalls oder erneut zu begehen. Das Strafen war damit für Merian nicht nur das Ausüben einer bestimmten Handlung bzw. das »reine« Sanktionieren einer nicht erwünschten Tat oder Äusserung, sondern stellt auch in dem Sinne eine Praktik dar, als damit eine normative Aussage verbunden war, die nicht nur die zu bestrafende Person betraf, sondern im konkreten Fall eine ganze Schulklasse.

Merian unterschied zudem zwischen verschiedenen »Fehlern«, die nicht alle zu bestrafen waren. Grundsätzlich durften nur »Fehler«, die nicht als »Naturfehler « zu klassieren waren, das heisst Fehlverhalten, das nicht auf eine körperliche Schwäche oder ein Defizit zurückzuführen sei, bestraft werden. Wenn etwa Schulaufgaben wegen mangelnder Gedächtnisleistung nicht zur Zufriedenheit des Schulmeisters ausgeführt wurden oder die Schüler auf Fragen nicht die richtige Antwort gaben, dann war unter bestimmten Umständen von einer Bestrafung abzusehen. Konkret war dies etwa dann der Fall, wenn der Schüler oder die Schülerin die Aufgaben nicht aus Faulheit, sondern aus intellektuellem Unvermögen oder einer anderen nicht selbst verschuldeten Ursache nicht oder falsch erledigt hatte. Gründete das Nichtwissen allerdings auf Faulheit, dann lag ein moralisches Fehlverhalten vor, das zu bestrafen war. ${ }^{38}$

Merian unterschied nicht nur verschiedene Fehler, Unterschiede gab es auch bei der konkreten Strafpraxis, wobei diese nicht primär und auch nicht ausschliesslich aus Körperstrafen bestand. Grundsätzlich sollte zuerst mit Worten bestraft werden, wobei auch hier darauf zu achten war, dass strafende Worte nicht im Affekt ausgesprochen wurden. ${ }^{39}$ Ausführlich wurde zudem darauf verwiesen, dass und vor allem auch wie die Einsicht in die "pädagogische Notwendigkeit« der Strafe beim zu bestrafenden Kind hervorzurufen sei. "Stellet ihm vor, wie ihr es ungerne thut; es euch aber zwinge, weil es so gütigen Ermahnungen und Warnungen nicht gefolget habe; wie ihr es vor Gott zu verantworten hättet, wenn ihr das Böse dulden, und nicht strafen würdet; und wie es sein gröstes Unglück wäre, wenn ihr es ungestraft lassen würdet.$^{40}$ Strafen wurde als (religiöse) Pflicht charakterisiert, die schlimmeres Fehlverhalten verhindere, wobei deutlich zwischen einer Zeit für den Unterricht und einer Zeit für die Bestrafung getrennt wurde. Bestrafungen hatten immer erst nach dem Unterricht zu erfolgen, wobei auch darauf zu achten war, diese »unpartheyisch $\aleph^{41}$ auszuführen. Gegen eine reale oder diskursiv als real konstruierte Strafpraxis, die von Merian als ungerecht

\footnotetext{
38 Ebd.

39 Ebd., S. 48.

40 Ebd.

41 Ebd., S. 49.
} 
etikettiert wurde, zeigte der Leitfaden alternative Handlungsmöglichkeiten auf, wobei eine solche Beschreibung auch als normsetzende Praktik zu verstehen ist.

Trotz der zahlreichen Gemeinsamkeiten in den beiden Leitfäden von Brünings und Merian werden in diesen Publikationen auch unterschiedliche Strafverständnisse sichtbar. Die beiden Anleitungen teilten zweifellos die Überzeugung, dass zwischen »moralischen « und "andern« Fehlern zu unterscheiden sei, wobei nur die »moralischen« Fehler zu bestrafen waren. Welches Verhalten allerdings als "moralisches« Fehlverhalten $\mathrm{zu}$ bewerten war sowie die Frage, ob eine grundsätzliche Notwendigkeit für Strafen bestehe, wurde unterschiedlich eingeschätzt. Übereinstimmung wiederum herrschte in der Betonung der Trennung von Unterricht und Bestrafung sowie in den verschiedenen Hinweisen zur Körperstrafe bzw. den damit verbundenen Ermahnungen, nicht im Affekt und nicht auf empfindliche Körperteile wie den Kopf zu schlagen. Das lässt darauf schliessen, dass diese Strafen nicht nur existierten, sondern dass sie vor allem auch als unpassend und als zu vermeidend beurteilt wurden. Strafen werden somit in diesen beiden Anleitungen als durchaus alltägliche historische Praxis des Schulmeisters dargestellt, die ohne grosses Nachdenken und als `kulturelle Selbstverständlichkeit a ausgeübt wurde. Entgegen diesen Gewohnheiten versuchten die Handreichungen die mit dieser Praxis verbundenen Normvorstellungen durch die Beschreibung von alternativen Handlungsmöglichkeiten zu verändern, wobei diese Alternativen nicht theoretisch oder normativ, sondern mit Verweis auf die eigene, erfolgreiche Praxis begründet wurden. Damit lässt sich die in den Handreichungen beschriebene Strafpraxis auch als normgebende Praktik verstehen mit dem Ziel, eine konkrete Praxis zu modifizieren.

\section{Felbigers und Crauers Methodenbuch}

Das Thema "Schulzucht « wurde auch in Felbigers und in Crauers Methodenbuch diskutiert, den beiden wesentlichen Handreichungen für Schulmeister in katholischen Regionen Mitteleuropas bzw. der Schweiz. Vergleichbar zur Brünings und Merian betonte Felbiger in seiner 1775 erstmals erschienenen Publikation die Stufenförmigkeit von Strafen, wobei er ein sehr differenziertes Stufensystem vorschlug. Dieses reichte von mündlicher Ermahnung bis zur körperlichen Bestrafung mit der Rute und schloss auch den Schulausschluss mit ein, eine Strafe, die dann anzuwenden war, wenn körperliche Züchtigung nicht zum gewünschten Erfolg führe. ${ }^{42}$ Am ausführlichsten diskutierte Felbiger die Sanktion von Fehlver-

42 Felbiger 1775, S. 450. Die einzelnen Strafen wurden bei Felbiger detailliert beschrieben, womit das Methodenbuch seinen Anspruch, eine ausführliche und auf die Praxis hin orientierte Handreichung für Lehrer und Schulbehörden zu sein, wohl am »praxistauglichsten« von al- 
halten durch »Strafen «, da hier in den Schulen »meistens [...] übertrieben « werde, ${ }^{43}$ womit auch Felbiger wie schon Brünings und Merian auf die Alltäglichkeit des Strafens hinweist. Auch Felbiger ging davon aus, dass körperliche oder geistige Fehler, die auf Unvermögen beruhten, nicht zu bestrafen seien. »Temperamentsfehler« hatten bei Felbiger ebenfalls keine Bestrafung zur Folge, wobei zwischen denjenigen Fehlern unterschieden wurde, die aus »Uibereilung« und denjenigen, die aus »Unbesonnenheit« resultierten. ${ }^{44}$ Während die der Hektik geschuldeten Fehler straflos blieben, waren Fehler aus Unbesonnenheit, die bei Brünings als Fehler der »Sekundärtugenden« oder als »Verhaltensfehler« bezeichnet wurden »Flüchtigkeit, Unachtsamkeit, Schläfrigkeit, und Langsamkeit«- ${ }^{45}$ durchaus zu bestrafen.

Ebenfalls nicht zu bestrafen waren Fehler, die ausserhalb der Schule bzw. der Unterrichtszeit vorgefallen waren, oder Fehler, für welche nicht die Schüler, sondern deren Eltern, das Gesinde oder weitere Mitglieder des Haushalts verantwortlich waren. Während die Autoren der verschiedenen Anleitungen darin übereinstimmten - falls dieser Fall überhaupt thematisiert wurde -, dass die Schülerinnen und Schüler nicht für Fehler anderer verantwortlich gemacht werden durften, war der Umgang mit Fehlern, die ausserhalb der Schule oder der konkreten Unterrichtszeit begangen wurden, durchaus unterschiedlich. Bei Merian etwa - und mit ihm bei allen der hier diskutierten, aus protestantischer Feder stammenden Anleitungen - wurden die Schulmeister daran erinnert, dass sich ihre Aufgabe nicht auf die Unterrichtszeit beschränke, im Gegenteil. Sie irrten, wenn sie davon ausgehen würden, dass sie ihrem »Berufe schon ein Genüge gethan« hätten, wenn sie auf die »Kinder in der Kirche und in der Schule sehet. Eure Aufsicht und Sorgfalt für dieselben gehet noch weiter «, ${ }^{46}$ ermahnte etwa Merian seine Leserschaft. Er sprach in diesem Kontext zwar nicht von Strafen, wies aber darauf hin, dass sich die Schulmeister sowohl um das Verhalten der Schülerinnen und Schüler zu Hause als auch auf »der Gasse« zu kümmern hätten und dass sie die Kinder vor allem auch »auf der Gasse« ansprechen und sie zu ihrem Tun befragen sollten, um sie "an die göttliche Gegenwart und Allwissenheit « zu erinnern. ${ }^{47}$ Der erziehende Aspekt des Schulmeisterdaseins, von dem Strafen ein Teil war, er-

len hier diskutierten Handreichungen umsetzte. Es werden nicht nur die verschiedenen Stufen der Strafen - von den Ermahnungen über die Warnungen, Drohungen, Verheissungen bis hin zu den eigentlichen Strafen - minutiös beschrieben, sondern es wird auch zu jeder Strafform genau aufgezeigt, bei welchem Vergehen konkret welche Strafe wie umzusetzen war.

43 Ebd., S. 456.

44 Ebd., S. 457.

45 Ebd.

46 Merian 1779, S. 52.

47 Ebd. 
streckte sich damit ausdrücklich nicht nur auf die Schule, sondern war Teil des lokalen Kontextes, der dem Schulmeister eine zentrale Rolle zusprach.

Einig waren sich die verschiedenen Autoren darin, dass Fehler, die schon wiederholt angemahnt worden waren, zu bestrafen seien. Dazu zählte Felbiger konkret die Verletzung der Schulgesetze, »Unehrerbietigkeit, Widerspänstigkeit, Ungehorsam gegen die Vorgesetzten«, »Sünden« und »sündhafte Gewohnheiten, wodurch Gott beleidiget « werde - Lügen, üble Nachrede, Fluchen, Schwören, Versäumen des Gottesdienstes oder grober Mutwillen - und vor allem auch »Fehler, woran das Herz Theil nimmt «. ${ }^{48}$ Ebenfalls zu bestrafen war die »Verharrung in dem Bösen«, vor allem auch, wenn damit die Gefahr verbunden war, andere Schüler zu diesem Verhalten zu motivieren oder wenn sie von diesem Verhalten betroffen waren.

Während Felbiger bei den körperlichen Strafen und mündlichen Ermahnungen ähnliche Überlegungen formulierte, wie sie schon bei Brünings und Merian $\mathrm{zu}$ lesen gewesen waren, unterschied sich seine Einschätzung der Scham als mögliche Strafe ${ }^{49}$ "Alle Beschimpfungen und ehrenrührische Beschämungen « waren auch für Felbiger nicht akzeptabel. ${ }^{50}$ "Dagegen sind in Schulen gewisse Beschämungen nicht nur erlaubt, sondern auch nothwendig: solche nämlich, die auf ein Vergehen so zu sagen ganz natürlich passen.$^{51}$ Die Beschämung als Strafpraxis war demnach dann legitim, wenn auch das Fehlverhalten Scham zur Folge hatte und in alttestamentarischer Manier Gleiches mit Gleichem vergolten werden konnte.

Wieder in Übereinstimmung mit den Anleitungen von Brünings und Merian waren auch für Felbiger das Strafen im Affekt und das Strafen während der Unterrichtszeit zu vermeiden. Im Unterschied zu den (protestantischen) Anleitungen vertrat Felbiger allerdings das Prinzip der Reue, das nicht nur zur Einsicht in begangene Fehler führe und helfe, diese in Zukunft zu vermeiden. Reue konnte für Felbiger auch ein Grund darstellen, eine Bestrafung nicht nur auf später $\mathrm{zu}$ verschieben, sondern ganz darauf zu verzichten. Dabei war darauf zu achten, dass das Nachsehen einer Strafe oder deren Minderung den »Strafwürdigen, als auch einer ganzen Schule den größten Schaden « zufügen könne. ${ }^{52}$ »Bitten, Weinen, und Heulen eines Strafwürdigen « galten deshalb niemals als hinreichender Grund, eine Strafe aufzuschieben oder gar auszusetzen. Auch wenn der Lehrer »in Gefahr stünde durch die Erlassung der Strafe« sein Ansehen an der Schule zu

\footnotetext{
48 Felbiger 1775, S. 459.

49 Vgl. Frevert 2017, S. 84ff.

50 Felbiger 1775, S. 461.

51 Ebd., S. 462.

52 Ebd., S. 464.
} 
verlieren, durfte auf die Strafe nicht verzichtet werden. ${ }^{53}$ Strafverzicht war nur dann möglich, wenn der Fehler erstmalig aufgetreten war.

In Felbigers Augen besonders gefährlich war zudem die Tendenz der Schulmeister, bei besonders fähigen, fleissigen und begabten Schülern gelegentlich auf eine eigentlich angemessene Bestrafung zu verzichten, wobei dieses Verhalten umso öfter auftrete, je »besser« die schulischen Leistungen des Schülers seien. Diesen Lehrern legte er nochmals die Berücksichtigung seiner Strafgrundsätze nahe und betonte, dass das eben beschriebene Verhalten nicht nur für den einzelnen, nicht bestraften Schüler, längerfristig nachteilig sei, sondern dass es sich dabei vor allem auch um eine »Ungerechtigkeit" handle, die er als das "wahre Verderben solcher Kinder« bezeichnete..$^{54}$ Für Felbiger war damit nicht nur das Strafen an sich anleitungsbedürftig, sondern auch der Verzicht auf eine Strafe erforderte klare Kriterien. Sowohl die Ausübung als auch das Aussetzen einer Handlung hatten Folgen für Einstellungen und Verhalten, die gemäss den Ansprüchen, welche diese Anleitungen zu erfüllen versprachen, möglichst in eine kausale Beziehung zu bringen waren. Strafen war für Felbiger nicht nur eine konkrete Tätigkeit, die überlegt, mit dem notwendigen Sachverstand und mit Augenmass auszuführen war, sondern sie war auch eine pädagogische Handlung, deren Unterlassung problematisch werden konnte, weil durch das Nichtausführen falsche Anreize geschaffen und unerwünschten Normvorstellungen Vorschub geleistet wurde. Strafen war aber auch eine Tätigkeit, die nicht von persönlichen Gefühlen beeinflusst werden durfte, die ausschliesslich auf Verhaltensänderungen zielte und die im Fall des Schulmeisters auf die Zeit und den Ort der Schule und des Unterrichts beschränkt zu bleiben hatte.

Den Felbiger'schen Vorgaben folgte auch Crauers Methodenbuch (1786) weitgehend, auch wenn Crauer seine Bemerkungen zur Schulzucht wesentlich knapper formulierte. Was hingegen bei Crauer nicht $\mathrm{zu}$ finden ist, sind Ausführungen zur Reue und zur Möglichkeit, Strafen aufzuschieben oder ganz auszusetzen. Inwiefern diese Abweichungen der Nähe zum protestantischen Kontext geschuldet waren - Crauer war 1765 ins Kloster St. Urban eingetreten, das im Grenzgebiet zum protestantischen Oberaargau liegt -, kann nur vermutet werden. Die erfolgreiche Verbreitung der im 19. Jahrhundert in der Lehrerbildung verwendeten Geschichten der Erziehung zeigt allerdings, dass sich in protestantisch geprägten pädagogischen Kontexten bestimmte pädagogische Handlungsformen und Ausbildungstraditionen herausbildeten, die auch im katholischen Kontext als "richtig« und »weiterführend« angesehen wurden, weil die damit verbundene Intention nicht als konfessionell, sondern als pädagogisch konnotiert verstanden wurde. Für die Geschichten der Pädagogik bedeutet das, dass das in ihnen

53 Ebd.

54 Ebd., S. 465. 
vertretene Modell der beispielhaften Erzieher, an deren Vorbildfunktion sich die angehenden Lehrpersonen moralisch bilden konnten, sich auch in katholischen Kontexten durchsetzte - einfach mit katholischen Erzieherfiguren anstelle der protestantischen Klassiker. ${ }^{55}$ Reue und Strafverzicht könnten demnach als "nichtpädagogische« Handlung verstanden worden sein und deshalb keine Handlungsoption darstellen.

Auch die Stufeneinteilung der Fehler fiel bei Crauer knapper aus. Detailliertere Ausführungen und Überlegungen fehlen, Strafen wurden vielmehr als Fakt diskutiert, deren grundsätzliche Berechtigung nicht infrage zu stellen war. Aber auch hier wird deutlich: »Der Schullehrer muss bey dem Strafen nichts anders vor Augen haben, als die Besserung der Schüler, und er muss sich dabey gerecht zeigen, und die Strafe niemals im Zorne vollziehen $"{ }^{56}$ Strafen war auch bei Crauer eine sehr ernsthafte Angelegenheit, die nicht gedankenlos auszuführen war, sondern in der entsprechenden Gesinnung und mit der richtigen Absicht sollte das mit ihr verbundene Ziel erreicht werden können.

\section{Johannes Büels Bemerkungen für Landschullehrer}

Während die bisher vorgestellten Anleitungen die Themen Strafe und Schulzucht immer in eigenen Kapiteln diskutierten, sind in Büels Bemerkungen für Landschullehrer (1792) die entsprechenden Ausführungen über die ganze Publikation verstreut, wobei Büel seine Abschnitte nicht mit Titeln versah, sondern durchnummerierte. Dieses Handbuch erweckt deshalb eher den Eindruck einer assoziativ gereihten Sammlung von ausformulierten Merksätzen, die dem Schulmeister konkrete Handlungsanweisungen vermitteln, ohne ihn mit allzu ausführlichen Begründungen oder theoretischen Erklärungen zu »belasten«. Ergänzt wurden diese insgesamt 129 Abschnitte mit »ein paar Worten über Aufklärung " ${ }^{57}$ sowie einem Anhang, bestehend aus Auszügen von Johann Georg Hamanns Fünf Hirtenbriefen das Schuldrama betreffend (1763) und Adolph Freiherr Knigges Über den Umgang mit Menschen (1788). Abgesehen von diesem Anhang sei seine Schrift, so Büel in der Vorrede, »keine Compilation aus andern Erziehungsschriften, sondern einzig die Frucht meiner Aufmerksamkeit und meines Nachdenkens ${ }^{58}$ das nicht durch die Lektüre anderer Schriften behindert worden sei, habe er sich doch »des Lesens aller Schriften über Erziehung und Schulen so lange gänzlich [...] enthalten, bis ich den Charakter der Kinder und die Beschaffenheit meines Berufs

55 Tröhler 2013, S. $21 \mathrm{ff}$.

56 Crauer1786, S. 60.

57 Büel 1792, S. 107f.

58 Ebd., S. 6. 
selbst hinreichend [...] studiert « habe. ${ }^{59}$ Seine Ausführungen beruhten demnach vollständig auf seiner eigenen Erfahrung bzw. seiner schulmeisterlichen Tätigkeit. »Seit mehr als einem Jahr«, so Büel dann allerdings weiter, »lese ich alle Erziehungsschriften«. Dabei habe er nicht nur gemerkt, »wie viel schon gesagtes ich wieder sagte « und was er alles ausgelassen habe. Er habe beispielsweise auch gelernt, dass es einen Unterschied zwischen öffentlicher und privater Erziehung gebe, dass Erziehung auf dem Land und in der Stadt nicht identisch seien und »dass die ganze Erziehungskunst auf gewissen allgemeinen Grundsäzen beruhe, aus welchen ein verständiger Lehrer das besondre und lokale sich abstrahieren müsse.$^{60}$ Es gibt in Büels Einschätzung also durchaus so etwas wie eine »Theorie« der Erziehung, die allerdings auf die lokalen Bedingungen anzupassen sei. Seine Ausführungen zum Entstehungsprozess seiner Handreichung machen aber vor allem auch die Einteilung des Textes in nummerierte Abschnitte nachvollziehbar, die nicht einen durchgehenden, systematisch aufgebauten Text bilden, der sich an einer bestimmten Reihenfolge von Themen orientiert, sondern sich eher als Gedankensammlung auf der Basis einer bestimmten Erziehungs- und Unterrichtspraxis präsentiert, die weder einer Systematik folgt noch Allgemeingültigkeit beansprucht, die über die Darstellung der eigenen Erfahrung hinausreicht.

Auf den ersten 30 Seiten seiner Bemerkungen formulierte Büel Beobachtungen und Empfehlungen, die auf das Verhalten des Schulmeisters gegenüber Schülern, Eltern und Behörden zielten. Anschliessend beschäftigte er sich mit grundsätzlichen Überlegungen zum unterrichtlichen Handwerk und thematisierte das Verhalten der Schüler, bevor er in der 38. Bemerkung explizit auf das Thema Strafen und auf den Charakter des Schulmeisters zu sprechen kommt. ${ }^{61}$ Strafen wird von Büel nicht als Handlung oder Tätigkeit gesehen, die in bestimmten Situationen auszuüben oder zu unterlassen sei. Strafen wird vielmehr als moralisches Problem diskutiert, das mit der anthropologischen Frage verbunden wird, ob das Kind "gut« sei. ${ }^{62}$ Von der Antwort auf diese Frage unabhängig ging auch er davon aus, dass »Strafen [...] in einer Schule seyn« müssen, »so lange man in derselben Fehler und Vergehungen der Schüler antrefen wird. In der physischen und moralischen Welt sind Strafen mit Vergehungen immer in Verbindung; so sey es auch in der Schule!.$^{63}$ Fehler und Strafen waren für Büel reale Tatsachen, an

59 Ebd., S.7.

60 Ebd., S. 8.

61 In der vorhergehenden Bemerkung weist Büel darauf hin, dass »der Verfasser von Lienhard und Gertrud«, Johann Heinrich Pestalozzi, über Landschulen viel »Wahres und Trefendes« sage, »aber auch hie und da etwas, das Ideal ist und bleiben wird« (ebd., S. 31). Inwiefern diese Bemerkung einen Zusammenhang zum nachfolgenden Abschnitt hat, der sich dem Thema Strafen widmet, bleibt unklar.

62 Ebd., S. 57.

63 Ebd., S. 31. 
deren Existenz auf keine Weise etwas zu ändern oder die gar anzuzweifeln war, sondern mit denen umgegangen werden musste, wofür er die Lehrer durch seine Handreichung befähigen wollte.

Wie die bisher diskutierten Handreichungen unterschied auch Büel verschiedene Fehler und Strafen, betonte die graduelle Angemessenheit der Strafe und warnte vor Bestrafungen im Zorn. ${ }^{64}$ Die konkrete Strafhandlung war zudem in der Regel nicht eine körperliche Bestrafung, sondern fand vielmehr auf der moralischen Ebene statt. Auch hier zeigen sich keine Unterschiede zwischen seinen Überlegungen und den Ausführungen in den anderen Handreichungen. Strafen ging in der Regel mit Begriffen wie Ehre und Schande einher, und der zu Bestrafende wurde beschämt oder an seiner Ehre gepackt. Büel behauptete sogar, dass er, "seit dem ich Schule halte«, sich nur »ein einzigesmal genöthiget« gesehen habe, »einen Schüler zu schlagen «. ${ }^{65}$ Die körperliche Strafe, das wird auch bei Büel deutlich, war zwar eine existierende Strafpraxis. Die Autoren der verschiedenen Handbücher stellten sich aber auch auf den Standpunkt, dass es sich dabei um eine Massnahme handle, deren Anwendung nur sehr zurückhaltend zu empfehlen sei, wobei diese Zurückhaltung nicht explizit als normative Vorgabe, sondern vielmehr implizit formuliert wurde, indem die eigene Strafpraxis, die vornehmlich ohne Körperstrafe auskomme, als Norm setzende Praktik vorgestellt wurde.

Auf die moralische Dimension des Strafens zielte auch Büels Hinweis, dass die Schüler mit Blicken zu den erwünschten Verhaltensformen geführt werden sollten bzw. konnten. ${ }^{66}$ Es sei zudem darauf zu achten, dass die Strafen die persönliche Beziehung des Schülers zum Schulmeister nicht beeinträchtigen würden, ${ }^{67}$ weshalb eine Strafe nicht einfach nur auszuführen war, sondern jeweils auf die positiven und negativen Folgen der zu bestrafenden Handlung hinzuweisen sei. Um die Bedeutung der persönlichen Beziehung zu betonen bzw. als Hinweis auf die Qualität der damit verbundenen Strafpraxis, wies Büel darauf hin, dass seine Schüler ihn sogar darauf aufmerksam machen würden, wenn sie ihre Hausaufgaben nicht erledigt hätten, ein »Fehler«, der normalerweise eine Bestrafung nach sich ziehe und deshalb eher verschwiegen werde. Aber: „Warum sollte das Kind lügen, wenn das Geständniß der Wahrheit ihm keine harten Verweise, keine Strafe zuzieht? «. ${ }^{68}$ Für ihn war zudem unklar, ob eher »Böses« zu bestrafen oder »Gutes« zu fördern sei. ${ }^{69}$ »Wenn es nothwendig ist, dass ein Lehrer mit Klugheit strafe, so ist es gewiss nicht weniger nothwendig, dass er mit Klugheit lobe und

\footnotetext{
64 Ebd., S. $31 f$.

65 Ebd., S. 32.

66 Ebd., S. 51.

67 Ebd., S. 93.

68 Ebd., S. 77.

69 Ebd., S. 96.
} 
belohne ${ }^{70}$ Strafe - und das ist bei Büel im Vergleich zu den bisherigen Handreichungen ein neues Argument - wurde in einem grösseren Kontext der Herstellung von moralisch richtigem Verhalten verortet, dessen Praxis zwischen Lob und Strafe oszillierte.

Unabhängig von den verschiedenen inhaltlichen Schwerpunktsetzungen der einzelnen Handreichungen kann als durchgängiges Thema festgehalten werden, dass es sich bei der Beschreibung und bei der praktischen Bearbeitung von Fehlern und Strafen um ein Handlungsfeld handelte, das in einem Bereich stattfand, der als zwischen den Tätigkeitsbereichen der Eltern und Schulmeister liegend zu bezeichnen ist, wobei für die protestantischen Autoren, so auch für Büel, feststand, dass die Aufgabe des Lehrers über den Ort und die Zeit des Unterrichts hinausweise. Deshalb sei darauf zu achten, dass es nicht zu Konflikten mit den Eltern komme ${ }^{71}$ wobei die Frage der Abgrenzung nicht nur die Strafpraxis betraf, sondern sämtliche Tätigkeiten des Schulmeisters, die auf das Verhalten und die normative, sittliche und moralische Orientierung des Schülers zielten. In diesem Sinn war die Beschreibung der Strafpraxis auch Teil einer Praktik, welche die verschiedenen institutionellen Einflusssphären von Familie und Schule gegeneinander abzugrenzen suchte.

\section{Strafen in der Stapfer-Enquête}

Die in den Anleitungen und Handreichungen formulierten Überlegungen zu Fehlern und der damit verbundenen Strafen legen die Vermutung nahe, dass das Thema "Strafe« auch in der Schweizer Schulumfrage von 1799 präsent ist. Diese Erwartung wird allerdings enttäuscht, was zumindest teilweise mit dem Aufbau des Fragebogens erklärt werden kann, der nach den "Lokalverhältnissen«, dem »Unterricht«, den »Personalverhältnissen« und den »ökonomischen Verhältnissen« fragte und damit auf die »äußere« und »formale« Organisation und Gestaltung von Schule und Unterricht zielte und weniger die moralisch-normativen Seiten des Unterrichtens und des Lehrerseins in den Blick nahm. Die Äusserungen zu Strafen und Fehlern finden sich denn auch ausschliesslich in den ergänzenden »Bemerkungen", bei denen die antwortenden Lehrerinnen und Lehrer die Möglichkeit hatten, eigene Themen und Wünsche zu notieren sowie Vorschläge zur Reform und Verbesserung der Schule anzufügen. Die weitgehende Nichtberücksichtigung des Themenfelds von Fehlern und Strafe kann allerdings auch als Hinweis darauf gelesen werden, dass aus der Sicht der Schulmeister hier kein grosser Reform- bzw. Handlungsbedarf bestand oder zumindest kein Diskussionsbedarf

70 Ebd., S. 115.

71 Ebd., S. 61. 
darüber mit der helvetischen Regierung, das heisst mit der politischen Behörde. Möglicherweise waren Fehler und Strafen für die Schulmeister um 1800 aber auch im Vergleich $\mathrm{zu}$ anderen in den Bemerkungen angesprochenen Sachverhalten nicht das dominante Thema ihrer schulischen Praxis. ${ }^{72}$ Nur insgesamt sieben Lehrer - von insgesamt 918 Personen, die Bemerkungen formuliert hatten äusserten sich zu diesem Themenfeld. ${ }^{73}$ Von sehr viel höherem Interesse für die Schulmeister waren etwa der unregelmässige Unterrichtsbesuch, der Umgang mit ihren weiteren Pflichten neben dem Schulehalten, die ökonomischen Verhältnisse - hier vor allem die bescheidene Bezahlung und der ungenügende Zustand des Schulhauses ${ }^{74}$ - sowie institutionell-organisatorische Themen wie die Dauer des Schuljahres, die Organisation der Schülerinnen und Schüler in Klassen oder die fehlenden oder zumindest ungenügenden Ausbildungsmöglichkeiten für Lehrpersonen. ${ }^{75}$

Die Kontexte, in denen Fehler und Strafen trotz diesen einschränkenden Vorbemerkungen dennoch thematisiert wurden, sind sehr unterschiedlich. Der 26-jährige Thomas Ganginer beispielsweise, Priester und Lehrer an der katholischen Schule von Lachen im Kanton Linth, merkte an, dass alle Bemühungen, die Schulen zu verbessern und diese »nüzliche Einrichtung [...] allen Kindern [...] teilhaft zu machen«, nur dann erreicht werden könnten, wenn die Regierung die Schulmeister »jn Betreff anwendbaren Straffen unterstüzung leisten«, für die »stets müßigen Gaßenbetleren andere Einrichtung trefen«, auf die Durchsetzung

72 Vgl. Fuchs 2015, S. 165. Ein ähnliches Bild-das Nichtthematisieren eines erwarteten Themaswird bei der Erwähnung bzw. Nichterwähnung des Namens Pestalozzi in der Stapfer-Enquête sichtbar. Entgegen der historischen Erzählung, die Pestalozzi zu einer der entscheidenden Figuren der helvetischen Reformbewegung und der Schulgeschichte stilisiert, wird er in der Stapfer-Enquête - wie eigentlich sämtliche, am Ende des Ancien Regimes verfügbaren pädagogischen Theorieangebote -, nicht oder kaum diskutiert (Fuchs 2015, S. 225f.; vgl. Horlacher 2014).

73 Eine Abfrage der Datenbank mit den Stichworten »Strafe« und »bestrafen « ergibt acht Treffer. Die französischsprachigen Texte wurden hier nicht berücksichtigt, da die diskutierten Anleitungen und Handreichungen auf das deutschsprachige Publikum gerichtet waren. Einer der Treffer, Lehrer Peter Badertscher aus dem bernischen Ebnit, spricht nicht die Strafpraxis im Unterricht an, sondern die finanziellen Folgen für die Schule, wenn die Eltern dafür bestraft werden, dass sie ihre Kinder nicht regelmässig zur Schule schickten. Das Problem sah Badertscher darin, dass die Kinder in diesem Fall überhaupt nicht mehr zur Schule gingen und er auf das entsprechende Schulgeld verzichten müsse (Stapfer-Enquête, Nr. 705). Das Thema Strafe in einem weiteren Verständnis wurde natürlich auch ohne die Verwendung dieser beiden Stichworte diskutiert, dies vor allem im Zusammenhang mit Disziplin. Da hier allerdings die Straf- und Fehlerpraktik im Zentrum steht, wurde ausschliesslich auf Nennungen fokussiert, die explizit die Strafe bzw. deren konkrete Anwendung ansprechen. 
der Schulpflicht bei den Eltern geachtet werde und Bemühungen unternommen würden, »gutte Schuhl Männer zu bekommen « ${ }^{76}$ Für Ganginer war die obrigkeitliche Unterstützung als formale Absicherung des "zulässigen« oder »angemessenen« Strafens eine der vier Hauptforderungen auf dem Weg zu einer Verbesserung des Schulwesens. Dass diese Forderung nicht nur an erster Stelle formuliert, sondern auch neben die Forderung nach qualitativ »besseren « Lehrpersonen gestellt wurde - eines der Hauptanliegen aller mit der Reform und Verbesserung des Schulwesens beteiligten Akteure -, unterstreicht die Bedeutung, welche das Strafen zumindest für den Schulmeister Ganginer in seiner alltäglichen Handlungsweise hatte.

Teilweise ähnliche Überlegungen formulierte auch Christoph Sulzer, Pfarrer und Lehrer in Brunnadern, Kanton Säntis, der wünschte, dass »zwekmäßige Belohnungen, u. Straffen da« seien. Während Belohnungen und Ermunterungen vollständig fehlten - Sulzer dachte hier konkret an Bücherprämien, schönes Schreibpapier oder Lobsprüche -, würden Fehler ausschließlich mit Schlägen bestraft. In seinen Augen sollten Strafen »beschämend« und Belohnungen »ermunternd« sein. ${ }^{77}$ Ähnlich äusserte sich auch Bendicht Hofer aus Heimiswil, Kanton Bern, der sich »schikliche Belohnungen, und Straffen für Elteren und Kinder « wünschte. ${ }^{78}$ Diese Bemerkungen lassen eine im Vergleich zu den verschiedenen Anleitungen und Handreichungen unterschiedliche Strafpraxis sichtbar werden. Während die Autoren der Handreichungen körperliche Strafen eher als nur selten anzuwendendes Strafmittel propagierten, beschreibt Sulzer diese eher als übliche Handlungen, was nochmals darauf hinweist, dass die Beschreibung der eigenen Strafpraxis in den Anleitungen und Handreichungen auch als normgebende Praktik zu verstehen ist, da sie nicht nur eine reale oder erwünschte Strafpraxis beschreiben, sondern in und durch diese Beschreibung eine Praxis verändern wollten.

Jacob Grundlehner hingegen, 38 Jahre alt, Vater von sieben Kindern und seit 19 Jahren Lehrer in Amriswil, Kanton Thurgau, sprach das Thema Strafen nur ganz kurz und eher beiläufig an, als er sich zu den für einen guten Lehrer notwendigen Charaktereigenschaften äusserte. Die Schulmeister sollten nicht nur »eine gutte Erziehung genoßen« haben, sondern in dieser »Kunst auch selbst geübt« sein sowie eine "sanfte Gemüths-Art besizen, damit sie bey der Bestraffung der Boosheiten, und Lasterhaften Vergehungen der Kinder bedachtsam zu werke gehen«. Das sei besonders deshalb wichtig, um »nicht im Zorn und Hize einem Kinde einen gefährlichen Streich « zu versetzen, der dann bedauert werde. ${ }^{79}$ Der

\footnotetext{
76 Stapfer-Enquête, Nr. 448.

77 Ebd., Nr. 1181.

78 Ebd., Nr. 1374.

79 Ebd., Nr. 85.
} 
Lehrer sollte also nicht nur über eine gute Ausbildung verfügen, sondern vor allem auch Geduld und Augenmass haben. Wie schon in den verschiedenen Anleitungsbüchern warnte auch Grundlehner vor Affekthandlungen, die - so kann zumindest die häufige Warnung davor interpretiert werden - wenn auch nicht an der Tagesordnung, so doch eine einigermassen häufige Praxis waren; durchaus auch mit gravierenden körperlichen Folgen, sicher aber mit Gewissensbissen seitens der Schulmeister.

Die wohl ausführlichsten Überlegungen zu Strafen und Fehlern formulierte der Lehrer der reformierten Schule in Glarus Jacob Steinmüller und Vater von Johann Rudolf Steinmüller (1773-1835), zu dieser Zeit Erziehungsrat des Kantons Säntis. Steinmüllers Bemerkungen am Ende des Fragebogens waren vollständig der Bestrafung der »Fehler meiner Zöglinge« gewidmet, wobei auch er »Stecken und Rute (welch leztere [sic!] mir unerträglich wäre) [...] noch immer aus meiner Schule verbannet« habe. Anstelle der körperlichen Bestrafung versuche er, seine "Kinder theils durch Liebe zugewinnen theils durch Ehrbegierde zum Rechtthun anzufachen«. Auch Steinmüller bezog seine Strafpraxis auf die moralische Ebene. Die Herstellung der inneren Einsicht ins richtige Tun stand im Zentrum sowie die Frage, welche Hilfsmittel diesen Prozess der Einsicht am wirksamsten unterstützten. Dazu passt, dass durch "Scham« bestraft wurde, dass Strafen auf den folgenden Tag verschoben werden konnten, um wegen der damit verbundenen Wartezeit die Wirksamkeit der Strafe zu erhöhen, was sich insgesamt als erfolgreicher gezeigt habe als »derbe Schläge«. Um zu verhindern, dass »keine vergehungen ungeahndet vorbey gehen «, hatte Steinmüller zudem ein System von Aufsehern eingerichtet, die in seinem Auftrag bzw. in seinem Namen das Tun und Verhalten der Schüler beobachteten und Fehlverhalten meldeten, damit dieses bestraft werden konnte. Die Steinmüller'sche Strafpraxis war damit durchaus umfassend..$^{80}$

Vergleichbar zu den Handreichungen unterschied auch Steinmüller zwischen Fehlern aus »Unwissenheit« und Fehlern aus »vorsezlicher Bosheit«, wobei in seinen Augen erschwerend hinzukomme, dass die Schüler aus ganz unterschiedlichen Familien stammten und deshalb auch "von so verschiedener Erziechung" seien. ${ }^{81}$ Umso mehr sei darauf zu achten, dass den Schülern »die Schul zu keinem Joch« gemacht, sondern dass sie gern zur Schule gehen würden, was Steinmüller

80 Ebd., Nr. 559.

81 Die unterschiedliche Bestrafung von Fehlern war auch bei Bendicht Arn aus Kappelen, Kanton Bern, Thema, wobei es Fehler gebe wie »augenscheinlicher Mutwillen, Lügen und Verführung der kleineren«, die zwingend zu bestrafen seien. Gedächtnisfehler hingegen, »kindliche Flatterhaftigkeit oder auch der Versaümniße deren die Eltern Schuld haben «seien nicht zu bestrafen, da dies kontraproduktiv wirke. Zudem sei »die öfftere Einschärfung der Cerechtigkeit und Treü, und der Tadel deß Müßiggangs und eines schlechten unedlen Betragens [...] sehr heilsam« (ebd., Nr. 829). 
seine Schüler auch immer wieder explizit fragte. "Schon oft sezte ich sie auch auf die Prob, ob sie lieber Feyrtag halten, oder in die Schul kommen wollen? und mit vergnügen, wählten sie lezteres « ${ }^{82}$

Steinmüllers ausführliche Bemerkungen zu seiner Strafpraxis und den sie leitenden Überzeugungen decken sich weitgehend mit den in den verschiedenen Anleitungen sichtbar gewordenen Strafpraktiken. Steinmüllers Bemerkungen können auch in dem Sinne als Praktik verstanden werden, als sie eine erfolgreiche Praxis beschreiben, die in ihrer Verschriftlichung Vorbildfunktion zugesprochen wurde, wenn auch nicht für den praktisch tätigen Schulmeister, so doch für die, für die Schulen politisch Verantwortlichen. In dem Sinne hatte Steinmüllers Beschreibung seiner Strafpraxis einen anderen Adressatenkreis im Blick als die Anleitungen und Handreichungen für Schulmeister, die damit verbundene Praktik Normsetzung durch die Beschreibung der eigenen praktischen Erfahrung - war hingegen identisch.

\section{Die Praxis des Strafens als soziale Praktik}

Der Fokus auf Fehler und Strafen und die damit verbundenen Handlungen in den verschiedenen Anleitungen und Handreichungen für Schulmeister sowie in der Stapfer-Enquête hat eine durchaus vielfältige Strafpraxis in Schule und Unterricht zutage gefördert. Es liessen sich Themenbereiche und Handlungsformen identifizieren, die in allen Publikationen mehr oder weniger identisch diskutiert wurden, unabhängig davon, ob es sich um einen Leitfaden protestantischer oder katholischer Provenienz handelte und auch unabhängig davon, ob die Erfahrungen des Autors aus der Alten Eidgenossenschaft, dem Alten Reich oder dem Habsburgerreich stammten. Auch wenn die konfessionelle Herkunft der Leitfäden möglicherweise unterschiedliche Vorstellungen von Fehler und Strafen präformierte - vor allem der Hinweis auf Reue ist in diesem Zusammenhang ein auffälliger Unterschied -, so hatte die politische oder geografische Situierung der Fehler- und Strafpraxis doch darüber hinaus keine weitreichende Bedeutung für die hier diskutierte Fragestellung. Der Umgang mit körperlichen Strafen, die Frage nach der Angemessenheit der Bestrafung, der erzieherische Aspekt der Strafhandlung sowie die richtige Haltung sowohl der Strafenden als auch der zu bestrafenden Person stellten sich als die immer wiederkehrenden Themen der Diskussionen um Fehler und Strafe heraus.

Die verschiedenen Handreichungen und Anleitungen haben sich zudem in unterschiedlicher Detailliertheit darum bemüht, möglichst direkte Handlungsanleitungen oder -leitlinien zu formulieren, auf welche die Schulmeister bei Be- 
darf zurückgreifen konnten. Es ist auch deutlich geworden, dass die Anleitungen und Handreichungen das Thema Fehler und Strafen kaum auf der explizit normativen Ebene diskutierten, sondern vor allem auf der Ebene der Praxisanleitung, was mit der Textgattung und den damit verbundenen Ansprüchen der Autoren zusammenhängt. In sämtlichen Anleitungen wurde zudem mehr oder weniger explizit darauf hingewiesen, dass diese Publikationen keine theoretischen Abhandlungen, sondern aus der Praxis und für die Praxis entstanden seien, wobei die konkrete Beschreibung der strafenden Handlungen nicht als Abbild einer bestimmten Realität, sondern eher als absichtsvolle Formulierung der zugrundeliegenden Überzeugungen oder eben als Praktik zu verstehen ist. Auf welcher Basis oder aufgrund welcher Überlegungen die einzelnen Autoren bestimmte Strafformen ausübten und damit als sinnvoll oder effektiv empfahlen, wird in diesen Anleitungen und Handreichungen höchstens marginal diskutiert, in der Regel aber einfach mit dem »what worked«-Argument legitimiert. Die eigenen Erfahrungen und die als erfolgreich erlebte und beschriebene Praxis wurden als Modell gesetzt, wobei die Dignität der praktischen Erfahrung nicht zur Diskussion stand.

Trotz zahlreichen, seit dem 18. Jahrhundert immer wieder sichtbar werdenden Bemühungen, die Legitimität der eigenen Erfahrung für generelle Aussagen oder zur Theoriebildung in Zweifel zu ziehen bzw. deren Grenzen aufzuzeigen, ${ }^{83}$ hat diese Praktik nichts von ihrer Faszination und Wirksamkeit verloren. Eine Forschungsperspektive, welche Praktiken in den Blick nimmt, hat sich damit auch als interessanter Fokus erwiesen, einen Zugang zur Frage nach der anhaltenden Bedeutung von Ratgeberliteratur zu eröffnen. Die Beschreibungen der verschiedenen Strafpraxen und Strafpraktiken in den Anleitungen, Handbüchern und in den Bemerkungen der Stapfer-Enquête haben aber auch gezeigt, dass auch eine auf Praxis und Praktik fokussierte Perspektive keine historische Realität zu rekonstruieren vermag, sondern dass es auch hier im Wesentlichen darum geht, so gut wie möglich zu rekonstruieren, was gemeint war, wenn etwas gesagt bzw. getan wurde. Dieses Tun-als-Sagen kann allerdings sehr wohl als Teil von Praktiken untersucht werden, weil so auch Tätigkeiten und Wirkmechanismen in den Blick genommen werden können, die mit einer andern methodologischen Perspektive weniger oder überhaupt nicht sichtbar gemacht werden können. In dem Sinn ist eine auf Praktiken fokussierte Perspektive genauso fruchtbar und »nützlich" wie jede andere methodologische Perspektive, da es auch hier primär darum geht, Fragestellung, Quellen und Methoden in eine produktive Übereinstimmung zu

83 Die Grenzen einer auf der persönlichen Erfahrung beruhenden Ratgeberliteratur wurde nur wenige Jahre später von Johann Friedrich Herbart in seiner Allgemeinen Pädagogik (1806) kritisch diskutiert. Herbart wies darauf hin, dass die Erfahrungen einer einzelnen Person immer nur einen beschränkten Einblick in potenzielle Handlungsmöglichkeiten biete, weshalb er sich mit der Frage beschäftigte, wie diese empirischen Erfahrungen systematisch bearbeitet werden können (S. 7f.). 
bringen, wobei der Entscheid für den einen und damit auch gegen einen anderen Zugang immer auch eine Frage der subjektiven Präferenz ist. ${ }^{84}$

Der Entscheid für einen Zugang ist allerdings unabhängig von der subjektiven Präferenz davon abhängig, welche methodologischen Perspektiven mit welchen Fragestellungen und welchen Quellen interessante im Sinne von die Diskussion anregende oder weiterführende Ergebnisse hervorbringen können. Ein Fokus auf Praktiken ist im bildungshistorischen Kontext durchaus weiterführend, hilft er doch, Bildungsgeschichte von ihrer traditionellen Fokussiertheit auf Personen, Institutionen und politische Rahmenbedingungen zu lösen und eine kulturgeschichtlich orientierte Perspektive zu stärken, die Phänomene von Erziehung und Bildung in ihrer gesamten Breite wohl besser im Sinn von adäquater in den Blick zu nehmen vermag. Dies soll aber nicht als Plädoyer für einen Foucault'schen Diskursbegriff verstanden werden, der vollständig auf Subjekte in der Bildungsgeschichte verzichtet. Vielmehr ist es ein Hinweis darauf - und das sollte der Beitrag zeigen -, dass Personen, Institutionen und politische Rahmenbedingungen durchaus eine Rolle spielen, dass diese aber nicht als zentrale Akteure zu verstehen sind, um die herum Bildungsgeschichte zu erzählen ist, sondern dass sie Texte und Kontexte für eine Bildungsgeschichte darstellen, die inhaltlich bestimmte Fragen stellt und auf dieser Basis historische Sachverhalte in den Blick nimmt und damit danach fragt, was Phänomene und Ereignisse in der Zeit und für die Zeit bedeute(te)n.

\section{Quellen und Literatur}

\section{Quellen}

Büel, Johannes (1792): Bemerkungen für Landschullehrer und für Freunde derselben. Nürnberg.

Brünings, Gottfried Christian (1778): Anleitung zu fruchtbarer Lehrart in den niedern Schulen. Mannheim.

Crauer, Nivard (1786): Methodenbuch für die Lehrer der Normal-Stadt- und Landschulen in der Republik Solothurn. O.O.

Ephemeriden der Menschheit, oder Bibliothek der Sittenlehre, der Politik und der Gesezgebung, Erster Band (1780). Leipzig.

Felbiger, Johann Ignaz von (1775): Methodenbuch für Lehrer der deutschen Schulen in den kaiserlich-königlichen Erblanden. Wien.

Herbart, Johann Friedrich (1806): Allgemeine Pädagogik aus dem Zweck der Erziehung abgeleitet. Göttingen.

84 Pohlig 2008. 
[Merian, Emanuel] (1779): Anleitung für die Landschulmeister. Basel. Schulordnung (1766): Der Stadt Basel Schulordnung. O.O.

Stapfer-Enquête (1799): Edition der helvetischen Schulumfrage von 1799; www. stapferenquete.ch

\section{Literatur}

Alkemeyer, Thomas/Buschmann, Nikolaus/Michaeler, Matthias (2015): Kritik der Praxis. Plädoyer für eine subjektivierungstheoretische Erweiterung der Praxistheorien. In: Ders./Volker Schürmann/Jörg Volbers (Hg.): Praxis denken. Konzepte und Kritik. Wiesbaden, S. 25-50.

Benner, Dietrich (1987): Allgemeine Pädagogik. Eine systematisch-problemgeschichtliche Einführung in die Grundstruktur pädagogischen Denkens und Handelns. Weinheim, Basel.

Brühwiler, Ingrid (2014): Finanzierung des Bildungswesens in der Helvetischen Republik. Vielfalt - Entwicklungen - Herausforderungen. Bad Heilbrunn.

Carr, Edward Hallett (1961): What is history? Cambridge (UK).

Chartier, Roger (1992): Einleitung: Kulturgeschichte zwischen Repräsentationen und Praktiken. In: Chartier, Roger: Die unvollendete Vergangenheit. Geschichte und die Macht der Weltauslegung. Frankfurt a.M., S. 7-23.

Cuban, Larry (1984): How teachers taught: Constancy and change in American classrooms, 1880-1990. New York.

Derbolav, Josef (1975): Pädagogik und Politik. Eine systematisch-kritische Analyse ihrer Beziehungen: mit einem Anhang zur »Praxeologie«. Stuttgart.

Depaepe, Marc (2000a): Introduction. In: Ders. (Hg.): Order in progress. Everyday educational practice in Primary Schools Belgium, 1880-1970. Leuven, S. 9-17.

Depaepe, Marc (2000b): Developing a theoretical framework for the history of educational reality in the classroom. In: Ders. (Hg.): Order in progress. Everyday educational practice in Primary Schools Belgium, 1880-1970. Leuven, S. 19-53.

Dinges, Martin (1997): Normsetzung als Praxis? Oder: Warum werden die Normen zur Sachkultur und zum Verhalten so häufig wiederholt und was bedeutet dies für den Prozess der »Sozialdisziplinierung«? In: Gerhard Jaritz (Hg.): Norm und Praxis im Alltag des Mittelalters und der Frühen Neuzeit. Wien, S. 39-53.

Dinges, Martin (2002): Neue Kulturgeschichte. In: Joachim Eibach/Günther Lottes (Hg.): Kompass der Geschichtswissenschaft: ein Handbuch. Göttingen, S. 179-192.

Dross, Fritz (2006): Normale Praxis: Von neuen Konzepten in der Armenpflege, angestrengten Bemühungen zweier Medizinalräte und der erfolgreichen 
Nicht-Gründung eines Krankenhauses. In: Sebastian Schmidt/Jens Aspelmeier (Hg.): Norm und Praxis der Armenfürsorge in Spätmittelalter und früher Neuzeit. Stuttgart, S. 219-233.

Frevert, Ute (2017): Die Politik der Demütigung. Schauplätze von Macht und Ohnmacht. Frankfurt a.M.

Fuchs, Markus (2015): Lehrerinnen- und Lehrerperspektiven in der Helvetischen Republik. Bad Heilbrunn.

Herman, Frederik/Depaepe, Marc/Simon, Frank/Van Gorp, Angelo: Punishment as educational technology: A form of pedagogical inertia in schools? In: Paul Smeyers/Marc Depaepe (Hg.): Educational Research: Networks and Technologies. Dordrecht, S. 203-219.

Holenstein, André (2014): Reform und Rationalität. Die Enquêten in der Wissensund Verwaltungsgeschichte der Helvetischen Republik. In: Daniel Tröhler (Hg.): Volksschule um 1800. Studien im Umfeld der helvetischen Stapfer-Enquête 1799. Bad Heilbrunn, S. 13-32.

Horlacher, Rebekka (2014): Pestalozzi und die Lehrer um 1800. In: Daniel Tröhler (Hg.): Volksschule um 1800. Studien im Umfeld der Helvetischen Stapfer-Enquête 1799. Bad Heilbrunn, S. 135-148.

Horlacher, Rebekka (2018): Religion als Ausdruck kultureller Erwartungen an Schule. Religionsunterricht an Solothurner Schulen um 1800. In: Schweizerische Zeitschrift für Geschichte 2018, H. 3, S. 437-458.

Jaritz, Gerhard (1997): Norm und Praxis in Alltag und Sachkultur des Spätmittelalters: »Widerspruch« und »Entsprechung«. In: Ders. (Hg.): Norm und Praxis im Alltag des Mittelalters und der frühen Neuzeit. Wien, S. 7-19.

Mitterauer, Michael/Saurer, Edith (1993): Editorial. In: Historische Anthropologie. Kultur-Gesellschaft-Alltag 1, H. 1, S. 1-3.

Müller, Max (1995): Pädagogik und Erziehungswissenschaft. Der praxeologische Übergang bei Derbolav. Bern.

Pohlig, Matthias (2008): Geschmack und Urteilskraft. Historiker und die Theorie. In: Jens Hacke/Ders. (Hg.): Theorie in der Geschichtswissenschaft. Einblicke in die Praxis des historischen Forschens. Frankfurt a.M., New York, S. 25-39.

Popkewitz, Thomas S./Yates, Lyn/McCulloch, Gary/Westberg, Johannes/Piattoeva, Nelli/Cappelli, Gabriele/Head, Randolph C. (2018): The cult of facts, romanticizing the archive, and ignoring styles of reasoning: Delusive technologies of conducting historical research. In: Bildungsgeschichte. International Journal for the Historiography of Education 8, H. 2, S. 191-220.

Priem, Karin/König, Gudrun M./Casale, Rita (Hg.) (2012): Die Materialität der Erziehung. Kulturelle und soziale Aspekte pädagogischer Objekte. Weinheim, Basel. 
Schmidt, Sebastian/Aspelmeier, Jens (2006): Vorwort. In: Diess. (Hg.): Norm und Praxis der Armenfürsorge in Spätmittelalter und früher Neuzeit. Stuttgart, S. 7-14.

Skinner, Quentin (2002): Meaning and understanding in the history of ideas (1969). In: Ders.: Visions of Politics. Bd. I: Regarding Method. Cambridge (UK), S. 57-89.

Tröhler, Daniel (2013): Languages of Education. Protestant Legacies, National Identities, and Global Aspiration. New York. 